\title{
Design of a Framework for Modeling, Integration and Simulation of Physiological Models
}

\author{
E. Zeynep Erson, and M. Cenk Çavuşoğlu
}

\begin{abstract}
Modeling and simulation of physiological processes deal with the challenges of multiscale models in which coupling is very high within and among scales. Information technology approaches together with related analytical and computational tools will help to deal with these challenges. Physiological Model Simulation, Integration and Modeling Framework, Phy-SIM, provides the modeling environment which will help to cultivate various approaches to deal with the inherent problem of multiscale modeling of physiological systems. In this paper, we present the modular design of Phy-SIM. The proposed layered design of Phy-SIM, separates structure from function in physiological processes advocating modular thinking in developing and integrating physiological models. Moreover, the ontology based architecture will improve the modeling process by the mechanisms to attach anatomical and physiological ontological information to the models. The ultimate aim of the proposed approaches is to enhance the physiological model development and integration processes by providing the tools and mechanisms in Phy-SIM.
\end{abstract}

\section{INTRODUCTION}

Emergence of systems biology provided a comprehensive and integrative perspective to examine the structure and function at the cellular and organism levels of complex biological systems instead of focusing on the isolated parts [1]. However due to the complex nature of the physiological systems, development, integration of multiscale models and linking the layers stand as one of the challenges for the model developers [2]. In this paper, we present the design of the Physiological Model Simulation, Integration and Modeling Framework, Phy-SIM, which is an information technology framework with related analytical and computational tools that will facilitate development, integration and simulation of physiological models. Besides providing tools to develop physiological models, the strongest feature of the framework is providing the environment to aid the development of integration approaches.

Reusability, easy extension/modification are desirable attributes evolution both in nature and in software. Modularity brings and advantage to modifications in modules without changing intrinsic behaviors and so leading high reusability [3]. Both structural modularity in anatomy and in functionality for biological systems should be considered

E. Z. Erson is with the Department of Electrical Engineering and Computer Science, Case Western Reserve University, Cleveland, OH 44106, USA e-mail:eze@case.edu

M. C. Çavuşoğlu is with the Department of Electrical Engineering and Computer Science, Case Western Reserve University, Cleveland, OH 44106, USA e-mail: cavusoglu@case.edu

This work was supported in part by National Science Foundation under grants CNS-0423253, IIS-0805495, IIS-0905344 and US DoC under grant TOP-39-60-04003 when developing models of physiological processes as they simplify modeling, abstraction and enable robustness [4].

Based on these realizations, Phy-SIM proposes two levels of modularity, Structural Modularity and Functional Modularity, which are new perspectives toward multilevel and multiscale integration of physiological processes. PhySIM's approach tries to represent both the physiological functionality and the anatomical information modularly to facilitate comprehensive studies of the complex system.

\section{RELATED STUDIES}

There are various studies for building mathematical models for different levels of biological systems. In addition to the cellular level models, higher levels of modeling for organs and organ systems have been studied intensely such as heart [5], [6], [7], lung, kidney [8], cardiovascular system models [9], [10].

Besides the efforts to develop individual models, there are also several initiatives that try to create environments for researchers from various disciplines to achieve a collaborative environment and develop tools for integrative physiology research. Digital Human Project [11], Physiome [12] and Virtual Physiological Human Network of Excellence [13] are the examples of such efforts.

One of the successful studies in cell level modeling, with an integrative approach, is the BioSPICE Project, which provides a framework for modeling, simulating intra and inter-cell processes [14]. Physiome Project provides tools to enable integration with quantitative descriptions of relations among models and parameter sets to identify these relations. JSIM [15] a Java-based simulation system, SAPHIR as part of VPH [8] and SemSim [16] a semantic simulation framework for the integration of multiscale models of cardiac circulation are the examples of such tools.

\section{System DESCRIPTION OF PHy-SIM}

Phy-SIM is a modeling, integration and simulation environment for physiological processes from tissue level models up to organ-organism levels. Phy-SIM aims to achieve the following in the domain of multiscale physiological model development and simulation:(i) separation of structure from function,(ii) anatomical and physiological annotations,(iii) enhanced integration step,(iv) easy model sharing, extension and reusability.

A module is a structurally and functionally meaningful part of a system that can be separated from other components. Modularity is advantageous for model storing, sharing and reproducibility [17] and as stated in [18], modularity is a 


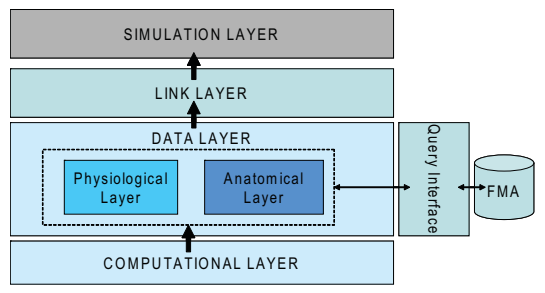

Fig. 1. Phy-SIM System Architecture: Phy-SIM has a layered design composed of Computational layer, Data layer, Link layer and Simulation Layer. The data layer is connected to the relational databases storing the anatomical ontology via a query engine interface.

desirable feature for the definition of physiological models, which usually is not properly implemented. To achieve structural and functional modularity, Phy-SIM is designed as a layered system, separating anatomical, physiological and computational models from the functionality as seen in Fig. 1. The separated functionality includes both the domain functionality which is the integration of these models as well as from the application specific functionality, simulation. Anatomical and physiological ontologies are used to annotate and modularize the physiological models. Using the functional and structural modularity together with the physiological and anatomical annotations, the integration of models will have a more systematic process in Phy-SIM.

From the perspective of model developers using PhySIM, possible use cases are (i) create/save/load mathematical models of physiological processes, (ii) annotate the models with anatomical and physiological ontology, save/load this information, (iii) load models of interest, perform queries on these models based on anatomical and physiological information and (iv) program various integration algorithms for models, perform the algorithm and test it. These use cases separates Phy-SIM from other biological simulation tools such as Matlab (Systems Biology Toolbox) [19], BioSPICE [14] and JSIM [15].

The four main layers in Phy-SIM which are designed to achieve the mentioned use cases are Computational layer, Data Layer, Link Layer and Simulation Layer.

\section{A. Computational Layer}

Mathematical models of the physiological processes show variations in concurrency and time constraints. Models of computations in the computational layer will provide the templates for different types of models with different constraints or requirements. Phy-SIM classifies the models of computation according to the ways they deal with concurrency and time concepts as: continuous time models, discrete time models and discrete event models [20]. Mathematical equations describing the dynamics of models, computational models (continuous time, discrete time or discrete event) and numerical integrators for continuous time models are defined at this layer. Based on the layered design, computational models implemented in this layer are independent of the physiological or anatomical elements in the data layer.

Every physiological variable defined in the data layer as described in the next section is assigned a mathematical model aggregating the equation of the model together with the computational model to solve the dynamics.

\section{B. Data Layer: Structural Modularity}

Data layer is responsible for the anatomical and physiological information component of the models. Anatomical layer, a sub-layer within the data layer, is designed to represent the anatomical structural information through the use of an ontology. Foundational Model of Anatomy (FMA) [21], [22], is used to represent the taxonomy and part-whole relations for the anatomical information. The FMA ontology will be accessible to the users at various steps of the model development and integration steps. Taxonomic representation will be easily accessible through a tree like presentation for steps such as annotation and querying (See Fig. 3).

Once the anatomical associations are bound to the physiological models, this information can be saved in an XML file which later can be loaded together with the models. This structured information binding will improve the sharing of models and increase the efficiency of collaborative works. Moreover the model developers will also benefit from the structured annotations during the integration processes. Application of the integration approaches are based on the structural information, such as, anatomical scales of models and physiological compatibility. Other anatomical information, such as, the part-whole relationships, constitutive relationships and spatial relationships will contribute to the integration of models. By the use of the querying interface and the anatomical associations, the developers will be able to view the models with these criteria. For instance, integrating a circulatory system model with an organ level model, will require to identify the physiological variables based on the anatomical associations such as the arterial supply of the organ and the constitutive relations of the arteries. In other words, standardized annotations of the models through the use of ontologies will provide the mechanism to analyze the models with different perspectives.

Physiological variables are defined based on their qualitative, quantitative and temporal attributes. Each physiological variable can have different temporal characteristic (single time or spanning a time course), can be scalar or vector valued and can be defined at a single point or over a field. Taxonomic organization of physiological variables are based on the type of information they carry and the information flow medium they are being used : (i) Circulatory physiological variable, (ii) Nervous Physiological Variable and (iii) Spatial Physiological Variable. Circulatory physiological variables are further specialized based on the type of information they carry. These variables are pressure, flow, volume, temperature, concentration variables and they determine the dynamics of the circulation.

The FMA ontology will be accessible through a querying interface to the developers. The latest version of FMA ontology in OWL representation is stored in a relational database (MySQL) and a querying engine is implemented. This engine is implemented in Java with the Jena/ARQ, a programming and querying framework for OWL. 
Based on the level of anatomical information available, developers can query the FMA ontology to retrieve the associated anatomical structure using the querying interface provided in Phy-SIM. Since the ontology provides a taxonomic and part-whole relationship, different level of anatomical structure will be used to annotate the models. For instance, if the model developer is working on a cardiopulmonary mechanics model, a physiological variable representing the pressure at the systemic circulation can be associated as vena cava, as systemic vein or just as vein depending on the detail of the model itself. The query interface will provide performing such queries.

The other sub-layer in the data layer is the physiological layer. There are some proposed ontologies for physiological processes which are in preliminary form, and are not as established or comprehensive as, for example, the FMA. Foundational Model of Physiology (FMP) [23], Ontology of Physics for Biology (OPB) [24] are the recent efforts for building a reference ontology of physical principles used in physiological processes. For the current implementation, Phy-SIM uses a high level ontology like representation for physiological information. However it should be noted that, with the modular design, integration of any physiological ontology into Phy-SIM architecture would be easy.

\section{Link Layer: Functional Modularity}

The mathematical models for physiological processes defining the current state of the whole system represent the regulation, control and modification of a physiological variables. A change in a physiological variable has a direct or indirect effect on processes determining other physiological variables in defining state of the system, like homeostasis. Based on this observation integration of physiological processes is conceptualized by the transfer, access or sharing of information among the models representing the processes, and is defined as information flow by the authors. Integration of multiscale processes can also be defined with information flow. If the processes to be integrated are from different scales, interpretation of information among scales is required before the information can carried among models. Link layer is the layer where the models are integrated based on the anatomical, physiological and computational information provided from the data and computational layers. The concept of a coupled dynamics among physiological variables is represented at this layer.

In the presented study, integration of physiological processes are grouped into two basic groups based on the scale and level of the anatomical and physiological structure that the processes take place. Vertical Integration refers to the integration of physiological processes via variables which carry information from different hierarchical levels of anatomical and physiological structures. Horizontal Integration is the type of integration that uses the modularity for the biological components within the same level. The separation of vertical and horizontal integration is also defined in VPH project [13].
Organs in a specific organ system or set of organs in different organ systems communicate in a horizontal organization through the circulatory and nervous systems. Use of circulatory and nervous systems as the main mediums and separating the structural information from the functionality is based on the analogy with computer networks, where the communication protocols are defined separately from the hardware of the network. As separating the layers in computer networks improves the development of elements in each layer, abstracting the circulatory and nervous systems and modularizing their functionality independent of the hardware of the network, i.e. anatomical and physiological organization, will enhance the development process of physiological models.

There are three basic architectural elements that handle the information flow and they are used to define every physiological model as a graph (Fig. 2). Component aggregates anatomical, physiological, computational information and corresponds to a node in the graph representation of the model. Semantic converter defines the links between the nodes. They also behave like a black box, where the information can be processed based on the integration approach (horizontal or vertical) which can be programmed by the modeler. Mediator is the control unit of the graph. The topology of the graph is perceived by the mediator, so that the ordering and cycle resolution type supervisions are done. The details of the functional modularity through information flow and example scenarios can be found in the other paper of Phy-SIM, submitted to EMBC 2010 [25].

\section{Simulation Layer}

Simulation layer handles the simulation of the presented models with the information from rest of the layers. Computational information defined in lower levels will be used in the simulation layer to decide the simulation specific parameters such as simulation step type, step size, simulation start and end times.

Simulation is an application specific functionality, hence it is defined at the highest level which accesses domain specific information in the lower levels. Every application will have a simulation manager, aggregating the mediator( $s)$, which is the access point to the domain specific layers.

Time step of a simulation has an effect on the accuracy of the models as well as on the performance of the whole framework, which are usually inversely proportional. Therefore based on the preference of the user, simulation time step should be a parameter that is adjustable. Determination of the time steps for the simulation depends on the individual update rates of models as well as the user defined parameters. The simulation time steps can be either variable or fixed.

\section{A Sample Model Integration Scenario}

In this section, we will show how the ontology based design will improve the integration of physiological models using example models obtained from Physiome repository.

First model is the cardiopulmonary mechanics model with four-chamber varying-elastance heart, pericardium, systemic 


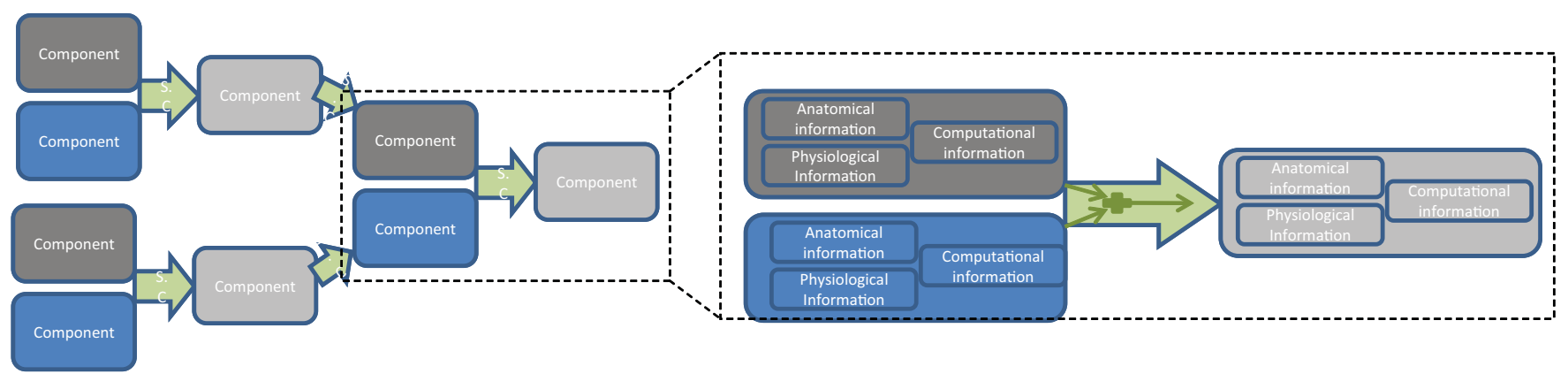

Fig. 2. Graph representation of the physiological model in the link layer. Semantic converters are the links in the graph representation which can also be programmed to perform computational operations in linking the physiological variables and models.

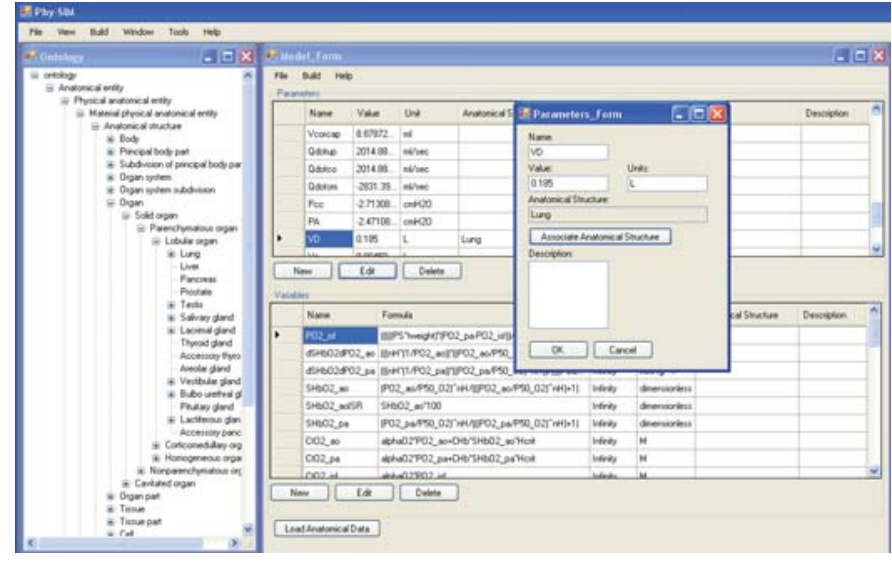

Fig. 3. The loaded models are seen on the right hand side of the frame. The parameters, variables together with the current values, units, mathematical equation describing the dynamics and the description are listed. The tree based view of the anatomical ontology is on the left of the frame, from which the selections can be made and the annotation can be performed.

circulation, pulmonary circulation and coronary circulation, baroreceptor and airway mechanics models. In the Physiome repository cardiopulmonary mechanics models are developed incrementally by adding sub-models, such as gas exchange.

For this demonstration we isolated the gas exchange model from the compound model, cardiopulmonary mechanics model with gas exchange. In the cardiopulmonary mechanics model; gas exchange model is coupled with the pulmonary circulation model, airway mechanics model, heart model, systemic circulation and coronary circulation through a set of variables. These variables are used to define the dynamics of oxygen and carbon dioxide pressure, concentration and saturation in the gas exchange model. In isolating the gas exchange model, we redefined the variables as parameters with their initial values and created a standalone model which can be simulated as is. As seen in Fig. 3, we have annotated these variables and saved the annotations in an XML file, which can later we loaded together with the model files ( $\mathrm{mml}$ files).

Anatomical and physiological annotations of the physiological variables will guide the integration process at this step. Based on the desired level of anatomical organization and physiological attribute, candidate physiological variables and associated models will be presented to the developer. Investigating the two models in detail without the use of any automated mechanism and trying to find out the integration points would be very inefficient for small models and not practicle for scale models . However, if one would be able to investigate the model based on their anatomical associations, and ask questions such as: What are the physiological variables/parameters which have the lung as an anatomical association? or What are the physiological variables/parameters associated with an organ which is part of lower respiratory tract? ; then investigation of the results of such an inquiry would provide a more systematic approach to perform the integration of the models over these variables. Similarly based on the annotations performed, the models can be analyzed with the physiological view and performing queries to handle questions such as: What are the physiological variables/parameters which are used to define a pressure?

Phy-SIM currently provides two different interfaces to perform queries on the models' anatomical attributes. One is based on an RDF query language, SPARQL and requires the user to type the query. This mechanism provides the flexibility to perform any kind of query. The other querying mechanism as seen in Fig. 4, helps the users to build the query by providing the tools. The user is given the options to select the model to perform the query on, the anatomical structure and the type of anatomical relation for that structure. Based on the selections made by the user, the SPARQL query is automatically generated, presented to the user and executed.

Besides determining the integration points for the models, the ontology based design will also help to determine the integration approach. In the case presented above, the parameter in the cardiopulmonary mechanics model and the variable in the airway mechanics model are at the same hierarchical organization in the anatomical ontology.

\section{DISCUSSION}

Phy-SIM is an information technology framework with related analytical and computational tools that will facilitate development, integration and simulation of physiological models. Besides providing tools to develop physiological models, the strongest feature of the framework is providing the environment to enhance the development of approaches 


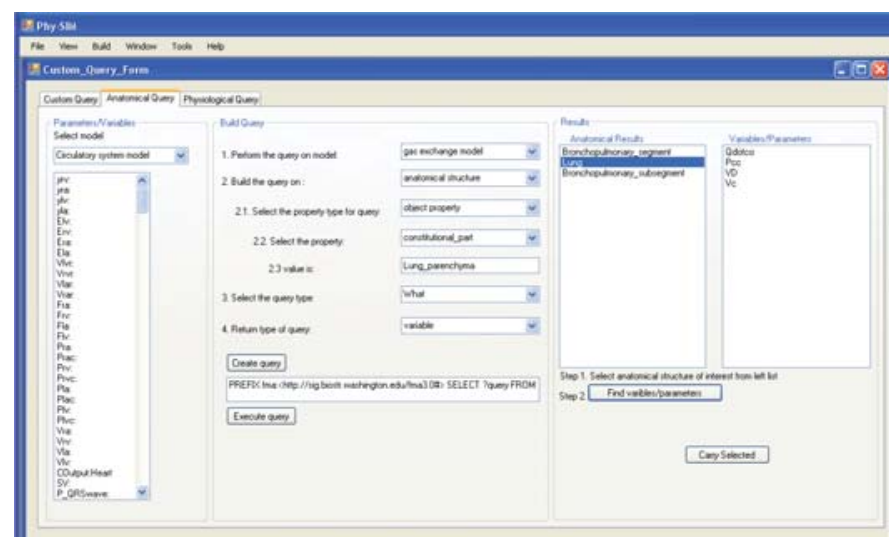

Fig. 4. The query interface: It provides a predefined list of selection to perform the most common type anatomical queries. The user can select which model to perform the query on, anatomical relation being queried and the matching variables in the selected model are returned. On the left of the frame, the facility to examine the variables and anatomical association of the models loaded is also provided as a reference to the users.

to achieve the linking of layers by the modular design principles proposed. However it is important to note some major open research problems in the domain of multiscale modeling and simulation for physiological process which are subject to much research.

Due to the complex nature of the human body, computation time is an inherent and open research problem [26]. There are various approaches to simulate multiscale models, such as brute force or model reduction. However in linking the layers, the trade-off between accuracy and complexity stands as an issue [27].

On the modeling side, as the coupling among physiological models is very high, integration of two models will often require managing this coupling at the model level. Even though Phy-SIM proposes mechanisms to handle this modeling process, identification of such a requirement and modeling it, is developer's responsibility.

Parametrization, standard representation of parameters with proper documentation is an other issue that has to be handled during model integration step. Since there is not any standard representation for the model assumptions, comparing and validating the compatibility of the models remain as an issue that has to be handled during model integration step.

Observing these inherent research problems in multiscale modeling and simulation, which are outside the scope of the present study, we propose a general simulation framework which would facilitate and allow the researchers to apply different methods and approaches to address these issues.

\section{REFERENCES}

[1] H. Kitano, "Systems biology: A brief overview," Science, vol. 295, pp. 1662-1664, 2002.

[2] D. Noble, "Systems biology and the heart," Biosystems, 2006.

[3] D. Floreano, S. Mitri, S. Magnenat, and L. Keller, "Evolutionary Conditions for the Emergence of Communication in Robots," Current Biology, vol. 17, pp. 514-519, 2007.

[4] M. E. Csete and J. C. Doyle, "Reverse engineering of biological complexity," Science, vol. 295, pp. 1664-1669, 2002.
[5] R. L. Winslow, D. F. Scollan, A. Holmes, C. K. Yung, and J. M. S Zhang J., "Electrophysiological modeling of cardiac ventricular function: From cell to organ," Annual Review of Biomedical Engineering, vol. 2, pp. 119-155, 2000.

[6] P. Kohl, D. Noble, R. L. Winslow, and P. J. Hunter, "Computational modelling of biological systems: tools and visions," Philosophical Transactions: Mathematical, Physical and Engineering Sciences, 2000.

[7] D. Noble, "Modeling the heart-from genes to cells to the whole organ," Science, 2002.

[8] S. R. Thomas, E. Abdulhay, P. Baconnier, J. Fontecave, J. P. Françoise, F. Guillaud, P. Hannaert, A. Hernãndez, V. Le Rolle, P. Maziere, F. Tahi, and F. Zehraoui, "Saphir - a multi-scale, multi-resolution modeling environment targeting blood pressure regulation and fluid homeostasis," in Proceedings of the 29th Annual International Conference of the IEEE EMBS, pp. 6648-6651, 2007.

[9] J. T. Ottesen, M. S. Olufsen, and J. K. Larsen, Applied Mathematical Models in Human Physiology. SIAM, 2004.

[10] A. I. Hernãndez, V. Le Rolle, A. Defontaine, and G. Carrault, "A multiformalism and multiresolution modelling environment: application to the cardiovascular system and its regulation," Physical and Engineering Sciences, pp. 4923-4940, December 2009.

[11] G. Higgins, B. Athey, J. Bassingthwaighte, J. Burgess, H. Champion, K. Cleary, P. Dev, J. Duncan, M. Hopmeier, D. Jenkins, C. Johnson, H. Kelly, R. Leitch, W. Lorensen, D. Metaxas, V. Spitzer, N. Vaidehi, K. Vosburgh, and R. Winslow, "Final report of the meeting "modeling \& simulation in medicine: Towards an integrated framework"," Computer Aided Surgery, pp. 32-39, 2001.

[12] "Physiome project, http://www.physiome.org/."

[13] "Virtual physiological human network of excellence." http://www.vphnoe.eu/.

[14] T. Garvey, L. P., C. Pedersen, D. Martin, and M. Johnson, "Biospice: access to the most current computational tools for biologists," Omics: A Journal of Integrative Biology, vol. 7, pp. 411-420, 2003.

[15] "Jsim, http://www.physiome.org/jsim/."

[16] M. L. Neal, J. H. Gennari, T. Arts, and D. Cook, "Advances in semantic representation for multiscale biosimulation: A case study in merging models," in Proceedings of the Pacific Symposium on Biocomputing, pp. $305-315,2009$.

[17] J. Bassingthwaighte, P. Hunter, and D. Noble, "The cardiac physiome: perspectives for the future," Experimental Physiology, 2009.

[18] P. Kohl and N. D., "Systems biology and the virtual physiological human," Molecular Systems Biology, 2009.

[19] H. Schmidt and M. Jirstrand, "Systems biology toolbox for matlab: A computational platform for research in systems biology," Bioinformatics Advance Access, vol. 22, 2005.

[20] C. Hylands, E. Lee, J. Liu, X. Liu, S. Neuendorffer, Y. Xiong, Y. Zhao, and H. Zheng, "Overview of the ptolemy project," tech. rep., Department of Electrical Engineering and Computer Science, University of California, Berkley, 2003.

[21] C. Rosse, L. G. Shapiro, and J. F. Brinkley, "The digital anatomist foundational model: Principles for defining and structuring its concept domain," in Proceedings, American Medical Informatics Association Fall Symposium, pp. 820-824, 1998.

[22] C. Rosse, J. L. V. Mejino, B. R. Modayur, R. M. Jakobovits, K. P Hinshaw, and J. F. Brinkley, "Motivation and organizational principles for anatomical knowledge representation: The digital anatomist symbolic knowledge base," Journal of the American Medical Informatics Association, 1998.

[23] D. L. Cook, J. L. V. Mejino, and C. Rosse, "Evolution of a foundational model of physiology: Symbolic representation for functional bioinformatics," in Proceedings, MedInfo, pp. 336-340, 2004.

[24] D. L. Cook, J. L. V. Mejino, M. L. Neal, and J. H. Gennari, "Bridging biological ontologies and biosimulation: The ontology of physics for biology," in In Proceedings, American Medical Informatics Association Fall Symposium, pp. 136-140, 2008.

[25] E. Z. Erson and M. C. Çavuşoğlu, "Functional modularity in integration of physiological system models with information flow." Submitted to International Conference of the IEEE Engineering in Medicine and Biology Society, EMBC 2010, 2010.

[26] J. B. Bassingthwaighte, H. J. Chizeck, and L. E. Atlas, "Strategies and tactics in multiscale modeling of cell-to-organ systems," in Proceedings of the IEEE, vol. 94, pp. 819-831, 2006.

[27] J. B. Bassingthwaighte and H. J. Chizeck, "The physiome projects and multiscale modeling," IEEE Signal Processing Magazine, 2008. 